diminished, as during the act of accommodation, the radii of curvature of the smalier lens changes more than that of the larger. Therefore the refraction of the smaller lens increases more as a result of accommodation than the larger, and the range of accommodation is correspondingly increased. The size of the lens must have something to do with its range of accommodation, and as $I$ understood the essayist, his inten tion is to raise that question in the minds of those present, so that they may bear it in mind, and, if possible, make due allowance for it in their clinical work. Oculists can not, for instance, demonstrate the exact power of the ciliary muscle, nor do they always take into account corneal imperfections. They presume there are cases in which there are certain differences in muscular power, etc., and they try as nearly as possible to make allowance for them in their work. Their allowances are based upon hypotheses, and somewhat upon clinical experience.

While the paper is not in the nature of an exact demonstration, it offers a valuable suggestion, and one which in future will, perhaps, influence the work of the ophthalmologist to the betterment of the condition of the patient. We all know how unsatisfactory the work of the ophthalmologist is in refraction. No one knows it so well as the ophthalmologist himself. On account of the lens and other parts of the eye being a living organism, instead of a mechanic apparatus, it is difficult for ophthalmologiste to make due allowance for the varied conditions and compensatory phenomena that are supposed to exist in it. But the little knowledge they have of these varied conditions aids them in arriving at more correct conclusions, and the more they add to this knowledge the better will be their work. The science of optics of the human eye is not yet per fected, and anything which leads the way to the betterment of this science should be hailed with pleasure by the profeseion. I wish, therefore, to thank Dr. Huizinga for his very valuable paper, and to express the hope that he will pursue his investigations and reduce his hypothesis to a scientific demonstration. Faraday did not demonstrate the principles of what is now known as electric faradization. He was only a prophet of what was to be in the future. His work was hypothetic, but was very valuable nevertheless.

Dr. Huizinga (closing the discussion)-Dr. Wilder spoke about asthenopia being due largely not only to the general condition of the patient, but to the condition of the ciliary and other muscles of the eye. I recall the case of one of my first patients treated years ago, which illustrated this forcibly. I refracted him perfectly, each eye separately, but he did not get along very well. He returned after two weeks for another consultation, and as an experiment I gave him some nux vomica in ascending doses, and at the end of a few weeks be returned, saying that he had thrown away his glasses and had entirely recovered. Since then I have followed this method of treat ment in a large number of cases of asthenopia of the ciliary muscle. (Here Dr. Huizinga explained the size of the lens by reference to charts.)

With reference to Dr. Gradle's last question, why the accommodation tires more easily in old than in young people, even when only a certain fraction of it is called into use, my answer is as follows: With the accommodation suspended, the lens is flattened and its fibers are forced to maintain an unnatural and cramped position. There is a constant effort on the part of these fibers to overcome this restraint. This tendency is in direct proportion to the elasticity of the lens. The result is that there is a constant tension exerted through the zonula upon the ciliary processes. This drawing upon these processes is a constant encouragement for the ciliary muscles to contract, and when only a slight enervation takes place the muscle immediately responds and is materially assisted by the great elasticity of the lens in young people. This assistance diminishes progressively with the age of the patient and the loss of elasticity of the lens, so that in an old person the muscle is obliged to do practically alone that which it formerly did with the assistance of the inherent elasticity of the lens. We would therefore expect this very delicate muscle to become more easily fatigued in old than in young persons.

\section{DISSIMILAR EYES.}

BY NORBURNE B. JENKINS, M.D. KNOXYILLE, TENN.

Many persons have considerable difference in the size and conformation of the eyes. The external measurements of one eye may differ from those of the other. One eye only may be perfect. Both eyes may be imperfectly formed, the imperfection of one eye differing in degree or in nature from that of its fellow. The combinations met with are numerous and varied. Occasionally one eye sees distinctly only distant objects, while the other is of service only for near vision. Often one eye is used for both near and distant vision, the other eye being used but little or not at all. A considerable difference sometimes exists until late in life without affecting the vision or comfort. On the other hand, slight differences frequently cause strabismus, amblyopia and asthenopia early in life.

The above condition is called "anisometropia." The reasons advanced for the frequent failures to correct it with lenses are herewith given. This one is taken from Fuchs" "Text-Book of Ophthalmology:" "The obvious course to pursue would seem to be to correct the anisometropia by ordering different glasses for the two eyes. Nevertheless, this measure in most cases proves impracticable. If the difference between the two glasses is somewhat great, the patients complain of an unpleasant sensation in the eyes, of vertigo, headache, etc., and lay the glasses aside. This fact is explained in the following way: Glasses alter not only the distinctness but also the size of objects. This is enlarged by convex and diminished by concave glasses, and the more so the stronger the glass is. With different glasses the retinal image of the same object is altered in size, more in one eye and less in the other; the images then no longer match one another, and can not be perfectly superimposed. We therefore prefer in anisometropia either to give the same glasses for both eyes, or to correct only one eye and place a plane glass before the other. In doing this we always have regard to the better eye, namely, the one which appears more efficient for the purpose in view (distant or near vision )."

In regard to this theory the following are offered: A convex lens magnifies objects and corrects hyperopia. A concave lens minifies objects and corrects myopia. A given person has in the right eye a myopia of $3 \mathrm{D}$, and in the left eye a hyperopia of $2 \mathrm{D}$. Without lenses an object appears larger to the myopic eye and smaller to the hyperopic eye than to an emmetropic eye. If perfectly fitting lenses are used, the retinal images of both the myopic and the hyperopic eye are made the same size as the retinal image of an emmetropic eye.

Of frequent occurrence are analogous cases to this: A given person has a total hyperopia of $4 \mathrm{D}$. in each eye. He wears with comfort, R.E.+ 1.75S.; L.E. + 3.25S., notwithstanding there is considerable difference in the power of such lenses, while the total error of refraction in either eye is the same.

Another explanation found in some of the textbooks on the eye is this: "Owing to the wide differerence in the refraction of the two eyes, only one eye, the less ametropic, is used for vision. The retina of the other eye has not been accustomed to clear images, consequently a glass which entirely corrects the error of refraction will not be tolerated. In these cases the less ametropic eye should be fully corrected and the more ametropic eye should be partially corrected, fitted with a plane glass, or given a glass of the same focus as that worn on the less ametropic eye."

The foregoing is probaby founded upon the observation of imperfectly refracted eyes in which, in addi. tion to myopia or hyperopia, there is astigmatism. It is probable that, with the methods laid down in the text-books, such errors of refraction can not be corrected to any degree of certainty. 
After cataract operations the best obtainable retinal perature, however, we find an important aid to diagimages are tolerated. Often persons with high de- nosis--that terrace-like rise from morning to evening. grees of ametropia pass many years of their lives If, in addition to the above systemic symptoms, the without the retinæ receiving clear images. When the temperature rises steadily from day to day and from errors of refraction are perfectly corrected, such per- morning to evening, and reaches 103 to 104 degrees sons experience no inconvenience from too distinct by the fourth day, there will be little reason to hesivision.

When the axis of the astigmatism is estimated to within three degrees, and the susceptible ametropia corrected to within $0.25 \mathrm{D}$., there is probably nothing to prevent an anisometrope using both eyes simultaneously for near or distant vision.

Note. Deformity ametropia, i. e, astigmatism of more than 3.5D., myopia of more than 7D., and hyperopia of more than $7 D$., have not been considered in the above article.

\section{TYPHOID FEVER.}

ITS DIAGNOSIS AND TREATMENT.

Read before the Baltimore County Medical Association.

$$
\text { BY H. S. JARRETT, M.D. }
$$

TOWSON, MD.

The subject of my paper is familiar to all, and I sincerely hope my humble efforts will at least be the means of bringing out a discussion of this very important subject, so that we may gather to ourselves even one point that will aid us in the future management of this dreaded and treacherous disease. My experience leads me to believe that we should do more than do nothing in typhoid fever cases, and treat the disease with antiseptic remedies. When called to a case of fever I always prescribe quinin and calomel, and if the fever does not yield to this treatment I consider the case as suspicious, and immediately change to an antiseptic, having already gotten the good effects of the calomel and preparing my patient for further treatment, which will be given later.

Obraztsow calls attention to the value of palpation of the ileum and mesenteric ganglia in the diagnosis and prognosis of typhoid fever. For the past four years he had observed, in examining the right iliac fossa, in cases of typhoid fever, that in 60 per cent. of the cases an intestinal loop can be felt beyond the cecum, about the size of the index finger, of varying consistence and at the level of which pain and gurgling were localized. Autopsy verified the author's belief that the loop was none other than the terminal point of the ileum, where it joined the cecum, and olinical observation has shown that the larger and more painful this loop the graver the prognosis of the case. The absence of this loop does not exclude typhoid fever.

As the palpation of these points is often rendered difficult by the sensitiveness and swollen condition of the large intestine, it will be seen that the greatest delicacy of touch is required. The diagnosis is often difficult, especially in children, as the symptoms are never as well marked as in adults.

You have feelings of malaise, which are often accompanied by a chill or chills. There is headache, dulness and listlessness, general soreness and chilliness, sometimes epistaxis, often diarrhea; sometimes moderate abdominal tympanites, with tenderness and gurgling in the right iliac fossa; and the tongue presents a thin, whitish coat, not extending to the tip and edges, which may be rather red. All or only some of these symptoms may be present, but are hardly pathognomonic, as many of them may be present in the early stage of other febrile affections. In the tem. tate as to the diagnosis. By this time the fecal discharges will begin to exhibit the characteristic light yellow color and pea-soup consistency. By the end of the first week the temperature will have reached the maximum point, which it is likely to hold during the disease, and the stage of invasion may be consid. ered as at an end.

From the end of the first week the temperature pursues a pretty steady course from the highest point, rising from morning to evening and falling from evening to morning from one to two and one-half degrees, the chart developing the characteristic zigzag appearance, the countenance becomes more dull, the eyes more suffused, the face more flushed, the tongue more coated, the intestinal discharges thinner and more frequent, the abdominal tenderuess and tympanites more marked, the skin dryer and congested. Both skin and breath exhale a peculiar odor, the pulse increases in frequency, rising, perhaps, from between 80 and 100 to between 100 and 120 , and is more in proportion to the temperature. The petechia or rose spots, slightly elevated above the surface and disappearing upon pressure, present themselves upon the abdomen and back. They usually appear, when present, about the seventh day. I remember some time since, during a discussion in our Society on this subject, great stress was laid on these spots. You will fail to diagnose many cases of typhoid fever if you depend on the spots. They are not always present, at least in my experience, and I believe they appear in only about 30 per cent. of the cases noted. In a report of 162 cases of typhoid fever in Porto Rico by Dr. Senn, army surgeon in the late war, he found the spots in only 37 cases.

We are indebted to Widal of Paris, who has given us the means of diagnosing a case of typhoid fever when diagnosis can not be based upon precise symptoms-the serum test-a drop of blood on your slide or visiting card, sealed and sent to your health board. The urine should always be examined for traces of albumin, and microscopically. for the bacilli, when possible. We all know the importance of good nursing and careful feeding in these cases. I invariably insist upon a definite amount of milk, given at stated intervals, as follows: Three ounces of cow's milk every two hours, giving one and one-half ounces of beef tea or broth alternately. I continue this dietetic treatment throughout the disease until the fever becomes normal in the evening, when I commence to increase the diet gradually. I do not give alcohol in any form, unless called for by the condition of the pulse.

Osler says that at least 75 per cent. of enteric fever patients recover under any and all forms of treat. ment, and even without the good nursing and regulated diet upon which we lay so much stress; and medicines are not, as a rule, indicated. No known drug shortens by a day the course of the fever. No method of specific treatment or of antisepsis of the bowels has yet passed beyond the stage of primary laudation. An impression has gone forth throughout the whole profession that all that could be done in the management of these cases was to regulate the 